\title{
Tamoxifen Pharmacogenetics and Metabolism: Results From the Prospective CYPTAM Study
}

Anabel Sanchez-Spitman, PharmD ${ }^{1}$; Vincent Dezentjé, MD, PhD²; Jesse Swen, PharmD, PhD ${ }^{1}$; Dirk Jan A.R. Moes, PharmD, PhD ${ }^{1}$; Stefan Böhringer, MD, PhD ${ }^{1}$; Erdogan Batman, MD³; Edith van Druten, MSN ${ }^{4}$; Carolien Smorenburg, MD, PhD ${ }^{2,5}$; Aart van Bochove, MD; Anneke Zeillemaker, MD, PhD7; Lynn Jongen, $\mathrm{MSc}^{8}$; Maartje Los, MD, PhD ${ }^{9}$; Patrick Neven, MD, PhD ${ }^{10}$; Hans Gelderblom, MD, PhD ${ }^{1}$; and Henk-Jan Guchelaar, PharmD, PhD ${ }^{1}$

PURPOSE Tamoxifen is widely prescribed as adjuvant therapy in patients with early-stage breast cancer. It has been postulated that concentrations of endoxifen, the active metabolite of tamoxifen, are a better predictor of tamoxifen efficacy than CYP2D6 genotypes. Although in a retrospective study, an endoxifen threshold of 5.9 $\mathrm{ng} / \mathrm{mL}$ for efficacy was described, confirmation based on prospective studies is lacking. The objective of the prospective CYPTAM (The Netherlands National Trial Register: NTR1509) study was to associate endoxifen concentrations and CYP2D6genotypes with clinical outcome in patients with early-stage breast cancer receiving tamoxifen.

PATIENTS AND METHODS From February 2008 to December 2010, patients with breast cancer treated with adjuvant tamoxifen were included. Patients could be enrolled up to a maximum of 12 months after tamoxifen initiation. Blood samples were retrieved for CYP2D6 genotyping and endoxifen measurements by Amplichip (Roche Diagnostics, Indianapolis, IN) and high-performance liquid chromatography-tandem mass spectrometry, respectively. Endoxifen concentrations were analyzed as a continuous variable, classifying patients into quartiles and using an endoxifen threshold of $5.9 \mathrm{ng} / \mathrm{mL}$. Endoxifen concentrations and CYP2D6 genotypes were associated with relapse-free survival (censored at the time of tamoxifen discontinuation; RFSt) by Cox regression analysis.

RESULTS A total of 667 pre- and postmenopausal patients were enrolled and had received tamoxifen for a median time of 0.37 years (range, 0.23 to 0.6 years) before study entry. No association was found between endoxifen concentrations and RFSt (adjusted hazard ratio, $0.991 ; 95 \% \mathrm{Cl}, 0.946$ to $1.038 ; P=.691$ ). Also, neither categorizing endoxifen concentrations into quartiles nor using $5.9 \mathrm{ng} / \mathrm{mL}$ as threshold altered these results. In addition, no association was found between CYP2D6 genotype and RFSt (adjusted hazard ratio, 0.929; $95 \% \mathrm{Cl}, 0.525$ to $1.642 ; P=.799$ ).

CONCLUSION This prospective clinical study shows no association between endoxifen concentrations or CYP2D6

ASSOCIATED

CONTENT

Appendix

Data Supplements

Podcast by Dr Stearns

Author affiliations

and support

information (if

applicable) appear

at the end of this

article.

Accepted on

December 6, 2018

and published at jco.

org on January 24,

2019: DOI https://doi.

org/10.1200/JC0.18.

00307

Clinical trial

information:

Netherlands Trial

Register NTR1509. genotypes and clinical outcome in patients with early-stage breast cancer receiving adjuvant tamoxifen.

J Clin Oncol 37. ๑ 2019 by American Society of Clinical Oncology

\section{INTRODUCTION}

In estrogen receptor-positive tumors, to block estrogen, endocrine therapy is indicated..$^{1,2}$ Tamoxifen and aromatase inhibitors (Als) are the pillars of endocrine therapy. In the adjuvant setting, tamoxifen can be prescribed as monotherapy or followed by a subsequent switch to an $\mathrm{Al}$ after 2 to 3 years of tamoxifen treatment. ${ }^{1-3}$ Initially, tamoxifen is metabolized into 4hydroxy-tamoxifen and N-desmethyl-tamoxifen (NDMtamoxifen), followed by a biotransformation into the most active metabolite, endoxifen (Fig 1). ${ }^{4}$ Also, CYP2D6 is considered the rate-limiting enzyme of the conversion of $\mathrm{N}$-desmethyl-tamoxifen into endoxifen. However, only $23 \%$ to $29 \%$ of endoxifen concentration intervariability is explained by genetic polymorphisms in CYP2D6 genotypes. ${ }^{5,6}$
In the study by Goetz et al, ${ }^{7}$ the authors described a statistically significant worsening of survival outcomes for patients with breast cancer with CYP2D6*4*4 genotype receiving tamoxifen compared with those of other CYP2D6 genotypes. Since then, many studies have been performed to confirm these findings, but conflicting results have been reported. Because CYP2D6 genotypes only partially explain the interpatient variability of tamoxifen ${ }^{8}$ and endoxifen concentrations, ${ }^{5,6}$ attention has been focused on endoxifen concentrations as a predictor for tamoxifen therapy. As a result of their decreased CYP2D6 activity, poor metabolizers (PMs) and intermediate metabolizers (IMs) have lower mean endoxifen concentrations compared with extensive metabolizers $(E M s) .8,9$ Studies performed in PMs and IMs treated with 


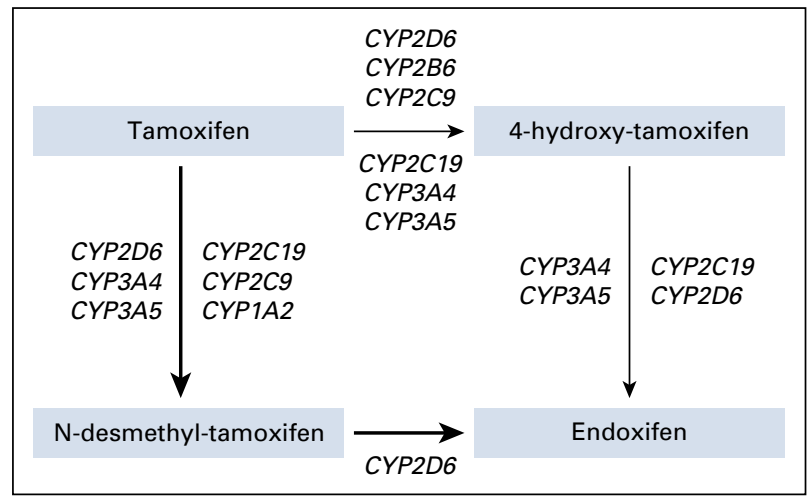

FIG 1. Metabolization of tamoxifen.

increased tamoxifen doses demonstrated that higher endoxifen concentrations were reached, without increasing short-term toxicity or adverse effects. ${ }^{10,11}$

Madlensky et al ${ }^{12}$ suggested a threshold of $5.9 \mathrm{ng} / \mathrm{mL}$ (15.8 $n M)$, above of which a $26 \%$ lower recurrence rate was described (hazard ratio [HR], 0.74; 95\% $\mathrm{Cl}, 0.55$ to 1.00 ). However, this threshold has been questioned, because the WHEL (Women's Healthy Eating and Living) study, from which these results were obtained, was not designed to determine such a threshold. Similarly, Saladores et $\mathrm{al}^{13}$ described another threshold of $5.2 \mathrm{ng} / \mathrm{mL}$ for premenopausal women, whereas Helland et $\mathrm{al}^{14}$ reported a threshold of $3.36 \mathrm{ng} / \mathrm{mL}$.

In several publications, the need for a prospective clinical study specifically designed to explore the relationship between endoxifen concentrations, CYP2D6 genotypes, and clinical outcome has been underlined. ${ }^{15}$ Therefore, the prospective CYPTAM (The Netherlands National Trial Register: NTR1509) study was initiated to properly address the question of whether CYP2D6 genotypes and endoxifen concentrations are related to clinical outcome in patients with early-stage breast cancer receiving adjuvant tamoxifen therapy. ${ }^{16}$ In Figure 2, a CONSORT diagram of the CYPTAM study is shown. The aim of this study was determine the association between CYP2D 6 genotypes or endoxifen concentrations and clinical outcome.

\section{PATIENTS AND METHODS}

\section{Study Design and Study Population}

From February 2008 to December 2010, women diagnosed with early-stage breast cancer receiving adjuvant tamoxifen were invited to participate in this multicenter prospective study executed in the Netherlands and Belgium. Pre- and postmenopausal patients who were at least 18 years of age and receiving treatment with the standard dose of $20 \mathrm{mg}$ of tamoxifen up to 12 months after initiating tamoxifen treatment were eligible for enrollment. The CYPTAM study was approved by the institutional review board of the Leiden University Medical Center in Leiden, the Netherlands, and recorded in the Netherlands Trial Registry. ${ }^{16}$ All the enrolled patients provided written informed consent. Individuals with a history of a previous malignancy within the last 5 years were excluded, with the exception of patients appropriately treated for an in situ cervix carcinoma or basal cell carcinoma. Serum and whole-blood samples were collected for measurements of tamoxifen and its metabolite trough levels and CYP2D6 genotyping, respectively, once steady-state drug concentration was reached. CYP2D6 genotyping was performed using the Amplichip CYP450 test (Roche Diagnostics, Indianapolis, IN) to test the major CYP2D6 alleles in DNA isolated from blood. According to their CYP2D6 genotypes, all patients were classified into predicted phenotypes, as described by Schroth et al, ${ }^{17}$ as ultra-rapid metabolizers (UMs), EMs, heterozygous EMs (hetEMs), IMs, and PMs. More detailed information is described in the Appendix (online only). At enrollment, clinical information was retrospectively collected and registered. From December 2016 until February 2017, information concerning survival outcomes was obtained from the medical records of the patients by professional data managers.

\section{Study Objectives and End Points}

Initially, the CYPTAM study was powered to detect a twofold worsening of 3-year relapse-free survival (RFS) of the combined group of PMs, IMs, and hetEMs, compared with EMs and UMs (HR, 2.0). The required sample size was estimated based on the studies of Goetz et $\mathrm{al}^{7}$ and Gonzalez-Santiago et al, ${ }^{19}$ in which HRs of 1.86 and 2.82 were reported, respectively. In total, 650 patients were needed to achieve $80 \%$ power at a .05 significance level.

However, since 2007, when the CYPTAM protocol was approved, more evidence suggesting endoxifen concentrations ( $v$ CYP2D6 genotypes only) to be a predictor for tamoxifen efficacy has been published. ${ }^{12-14}$ Therefore, before data analysis, the statistical analysis plan was amended, taking into account the role of endoxifen, and approved by the institutional review board.

The primary end point of RFS was defined as the time from study enrollment until locoregional or distant recurrence or second breast cancer. In case of a subsequent switch to an aromatase inhibitor (AI), patients were censored at the time of tamoxifen discontinuation (RFSt). The secondary end points were disease-free survival (DFS), complete RFS (RFSc), complete DFS (DFSc), and overall survival (OS). DFS was defined as the time from study enrollment until locoregional or distant recurrence or second breast cancer or death without recurrence. Patients who switched to an Al were censored at the time of tamoxifen discontinuation (DFSt). RFSc and DFSc differed from RFSt and DFSt in that the complete period of endocrine therapy (tamoxifen and $\mathrm{Al}$ use) until an event or loss to follow-up was included in the analysis. OS was defined as the time from study enrollment until death resulting from any cause. 


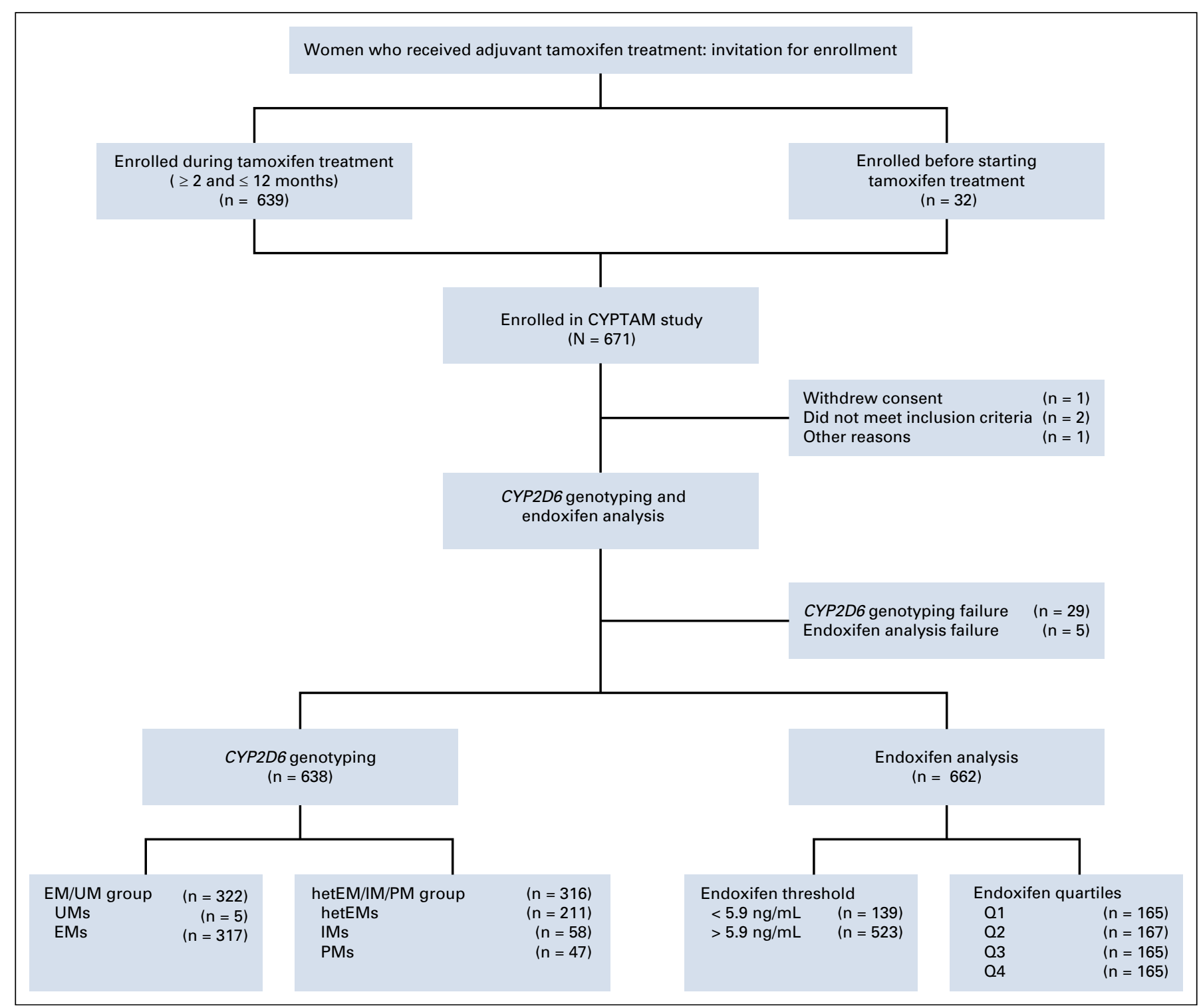

FIG 2. CONSORT diagram. EM, extensive metabolizer; hetEM, heterozygous extensive metabolizer; IM, intermediate metabolizer; PM, poor metabolizer; Q, quartile; UM, ultra-rapid metabolizer.

The primary objective was to determine the association of RFSt with endoxifen concentrations and CYP2D6 genotypes (UMs and EMs $v$ hetEMs, IMs, and PMs). The secondary objectives were to determine the relation between both endoxifen concentrations and CYP2D6 genotypes (UMs and EMs $v$ hetEMs, IMs, and PMs) and DFSt, RFSc, DFSc, and OS. This statistical analysis plan was designed as a gatekeeper analysis for the primary objective. Only in the case of an association between endoxifen and RFSt with $P<.05$ were the remaining objectives considered. Because of the multiple end points, the gatekeeper analysis was selected to avoid false-positive results by multiple testing ${ }^{20,21}$ and take into account the biologic and pharmacologic rationales. In addition, an exploratory analysis investigating the association between RFSt and endoxifen concentrations and CYP2D6 genotypes, accounting for the start of tamoxifen therapy, was performed.

\section{Statistical Methods}

To determine the association of RFSt with endoxifen concentrations and CYP2D6 genotypes, Cox regression and Kaplan-Meier analyses were performed. Endoxifen concentrations were primarily analyzed as a continuous variable in a Cox regression analysis. In addition, other approaches were explored using the endoxifen threshold concentration of $5.9 \mathrm{ng} / \mathrm{mL}^{12}$ Also, we examined categorizing patients into quartiles according to the reported endoxifen concentrations. Thereafter, Cox regression analysis and Kaplan-Meier methods were used to investigate any potential associations. All analyses were conducted using SPSS software (version 23.0; SPSS, 
Chicago, IL). Statistical significance was delimited as $P<$ .05. In the Appendix, the statistical methods used are more extensively reported.

\section{RESULTS}

\section{Baseline Characteristics, CYP2D6 Genotypes, Endoxifen Concentrations, Tamoxifen Therapy, and Follow-Up}

A total of 667 pre- and postmenopausal patients who were treated with adjuvant tamoxifen were enrolled in the CYPTAM study. Baseline characteristics of the enrolled patients suggested no differences across all the groups (Table 1), with the exception of progesterone receptor status in the comparison between CYP2D6-predicted phenotype groups. All CYP2D6 genotype distributions were in Hardy-Weinberg equilibrium, with the exception of CYP2D6*10 (Data Supplement). However, the genotype frequency of $C Y P 2 D 6^{*} 10$ in the study population was in accordance with previous evidence. ${ }^{22}$ Also, CYP2D6 phenotypes and endoxifen concentrations were significantly associated (Data Supplement).

In this study, the median follow-up was 6.4 years (range, 0.10 to 9.30 years), and the median tamoxifen therapy duration was 2.5 years (range, 0.25 to 7.96 years). Representing approximately $66 \%$ of the patients, the most frequent endocrine treatment strategy was the initial use of tamoxifen with a subsequent switch to an Al. At enrollment, $95.1 \%$ of the patients were already receiving adjuvant tamoxifen, whereas $4.9 \%$ of the enrolled patients started tamoxifen therapy at baseline. Before enrollment in the CYPTAM study, the median tamoxifen therapy duration for patients already receiving tamoxifen was 0.37 years (range, 0.23 to 0.60 years).

\section{Clinical Outcome and Endoxifen Concentrations}

No significant association was found between endoxifen concentrations, used as continuous variable, and clinical outcome (RFSt) in either univariable $(\mathrm{HR}, 0.989 ; 95 \% \mathrm{Cl}$, 0.945 to $1.035 ; P=.627$; Table 2 ) or multivariable analysis (adjusted HR, 0.991; 95\% Cl, 0.946 to $1.038 ; P=.691$; Table 2). Because no linear association or trend was observed, we explored other approaches. After dividing patients by endoxifen concentration below or above the suggested threshold of $5.9 \mathrm{ng} / \mathrm{mL}$, again no association was observed (adjusted HR, 1.538; 95\% Cl, 0.719 to 3.290; $P=$ .267; Table 2). In the same manner, after dividing patients according to their endoxifen concentrations into quartiles, no associations or trends were found when performing a log-rank test (quartiles: $P=.271$ ). These results were also verified by Cox regression analysis (Table 2). Similarly, the exploratory analysis investigating the association of RFSt with endoxifen concentrations from the start of tamoxifen therapy (instead of from actual study enrollment) did not alter these results (endoxifen concentrations [continuous variable]: adjusted $\mathrm{HR}, 0.986 ; 95 \% \mathrm{Cl}, 0.939$ to 1.035 ;
$P=.563$; endoxifen threshold of $5.9 \mathrm{ng} / \mathrm{mL}$ : adjusted $\mathrm{HR}, 1.422 ; 95 \% \mathrm{Cl}, 0.664$ to $3.043 ; P=.365$ ).

\section{Clinical Outcome and CYP2D6-Predicted Phenotypes}

Kaplan-Meier analysis did not reveal any differences in RFSt between UMs EMs versus hetEMs, IMs, and PMs (logrank $P=.944)$. In Figure 3, a Kaplan-Meier survival analysis is shown. Of note, an important decrease in the number of patients after the third year of treatment was observed, which is explained by the preplanned switch to an Al that took place in almost $66.0 \%$ of patients in the CYPTAM study. In line with the results noted in the log-rank test, Cox regression did not indicate significant differences in RFSt between CYP2D6 phenotype groups in either univariable (HR, 1.020; 95\% Cl, 0.589 to $1.767 ; P=.944$; Table 3) or multivariable analysis (adjusted HR, 0.929; 95\% Cl, 0.525 to $1.642 ; P=.799$; Table 3 ). In addition, the exploratory analysis investigating the association between RFSt and CYP2D6 genotype from the start of tamoxifen therapy (instead of from actual study enrollment) did not alter these findings (UMs and EMs $v$ hetEMs, IMs, and PMs: adjusted $\mathrm{HR}, 0.962 ; 95 \% \mathrm{Cl}, 0.545$ to $1.700 ; P=.894)$.

\section{Gatekeeper Statistical Analysis Plan: Secondary Objectives}

According to the gatekeeper analytic design chosen, no additional analyses of the secondary objectives were required, because we failed to find an association in the primary objective. However, we performed sensitivity analyses of the secondary objectives as an exploratory analysis. None of the secondary objectives were found to be statistically significant when analyzing the association between endoxifen concentrations or CYP2D6 genotypes and the different end points (RFSt, DFSt, RFSc, DFSc, and OS; Data Supplement). Menopausal status was analyzed by categorizing patients by age (premenopausal: age $\leq 45$ years; perimenopausal: age 45 to 55 years; postmenopausal: age $\geq 55$ years). Similarly, no significant differences were observed (Data Supplement).

\section{DISCUSSION}

In this first prospective study, to our knowledge, low-activity CYP2D6 genotypes and low endoxifen concentrations were not associated with a worse clinical survival outcome. In our opinion, these results are highly representative of clinical practice, because they illustrate the effect of tamoxifen on survival outcome based on the endocrine treatment strategy of switching to an Al after 2 or 3 years of tamoxifen treatment, widely used in the adjuvant setting in early-stage breast cancer. Also, the CYPTAM population had comparable survival and event rates to those observed in larger studies $^{23}$ and in clinical practice. ${ }^{24}$

A high number of clinical studies have assessed the putative association of low-activity CYP2D6 genotypes with tamoxifen efficacy. In an attempt to resolve this ongoing controversy, the International Tamoxifen Pharmacogenomics Consortium ${ }^{25}$ performed a meta-analysis with a 
TABLE 1. Baseline Characteristics of CYPTAM Patients

No. (\%)

CYP2D6-Predicted Phenotypes

\section{Characteristic}

Age at enrollment, years

\begin{tabular}{ll} 
Mean & 57.0 \\
SD & 11.5 \\
\hline Tumor stage &
\end{tabular}

\begin{tabular}{l}
$\mathrm{T} 1$ \\
$\mathrm{~T} 2$ \\
$\mathrm{~T} 3 / \mathrm{T} 4$ \\
\hline
\end{tabular}

Not specified

Nodal stage

NO

N1

N2

N3

Not specified

Histologic classification

Ductal adenocarcinoma

Lobular adenocarcinoma

Other

Not specified

Histologic grade

\begin{tabular}{lr}
\hline 1 & 43 \\
\hline 2 & 186 \\
\hline 3 & 90 \\
\hline Not specified & 3 \\
\hline Progesterone receptor status
\end{tabular}

Progesterone receptor status

$\begin{array}{lc}\text { Positive } & 255(79.2) \\ \text { Negative } & 58(18.0) \\ \text { Not specified } & 9(2.8)\end{array}$

HER2 receptor status

O+

$1+$

$2+$

$3+$

Not specified

FISH

163

$163(50.6)$
$142(44.1)$
$13(4.0)$

4 (1.2)

148 (46.0)

141 (43.8)

$23(7.1)$

$8(2.5)$

$2(0.6)$

250 (77.6)

40 (12.4)

$30(9.3)$

$2(0.6)$

43 (13.4)

$186(57.8)$

90 (28.0)

$3(0.9)$

$\begin{array}{r}190(59.0) \\ 86(26.7) \\ 16(5.0) \\ 28(8.7) \\ \hline 2(0.6)\end{array}$

$2(0.6)$

30 (9.3)

$290(90.1)$

2 (0.6)

(continued on following page)

14 (4.4)

5 (1.6)

$152(48.1)$

117 (37.0)

$30(9.5)$

$16(5.1)$

$1(0.3)$

$239(75.6)$
$47(14.9)$
$29(9.2)$
$1(0.3)$

\begin{tabular}{c}
$44(13.9)$ \\
$173(54.7)$ \\
\hline $95(30.1)$ \\
\hline $4(1.3)$
\end{tabular}

$251(79.4)$
$64(20.3)$
$1(0.3)$

\begin{tabular}{c}
$192(60.8)$ \\
$80(25.3)$ \\
$19(6.0)$ \\
\hline $24(7.6)$ \\
$1(0.3)$ \\
\hline
\end{tabular}

Endoxifen Analysis, ng/mL

\begin{tabular}{lll}
\hline $\begin{array}{c}<.9 \\
(n=139)\end{array}$ & $\begin{array}{c}>\mathbf{5 . 9} \\
(\mathrm{n}=\mathbf{5 2 3})\end{array}$ & $\boldsymbol{P}^{*}$ \\
\hline & & .219 \\
\hline 55.3 & 56.7 & \\
\hline 10.3 & 11.3 & \\
\hline
\end{tabular}

.566

$\begin{array}{lll}175(55.4) & 77(55.4) & 277(53.0) \\ 122(38.6) & 61(43.9) & 211(40.3)\end{array}$

$1(0.7)$

$27(5.2)$

$0(0.0)$

$8(1.5)$

.188

68 (48.9)

$247(47.2)$

$52(37.4) \quad 213(40.7)$

15 (10.8)

42 (8.0)

4 (2.9)

19 (3.6)

$0(0.0)$

$2(0.4)$

.776

$105(75.5)$
$21(15.1)$
$13(9.4)$
$0(0.0)$

.872

$17(12.2)$

80 (57.6)

41 (29.5)

$1(0.7)$

.036

(20)

.899

$\begin{array}{cc}112(80.6) & 415(79.3) \\ 27(19.4) & 99(18.9) \\ 0(0.0) & 9(1.7)\end{array}$

$\begin{array}{cc} & 9(1.7) \\ 85(61.2) & 320(61.2) \\ 35(25.2) & 131(25.0) \\ 8(5.8) & 27(5.2) \\ 11(7.9) & 43(8.2) \\ 0(0.0) & 2(0.4)\end{array}$

\begin{tabular}{lcc} 
Positive (amplification) & $30(9.3)$ & $26(8.2)$ \\
Negative & $290(90.1)$ & $289(91.5)$ \\
\hline Not specified & $2(0.6)$ & $1(0.3)$ \\
\hline
\end{tabular}

.754

.754 
TABLE 1. Baseline Characteristics of CYPTAM Patients (continued)

No. (\%)

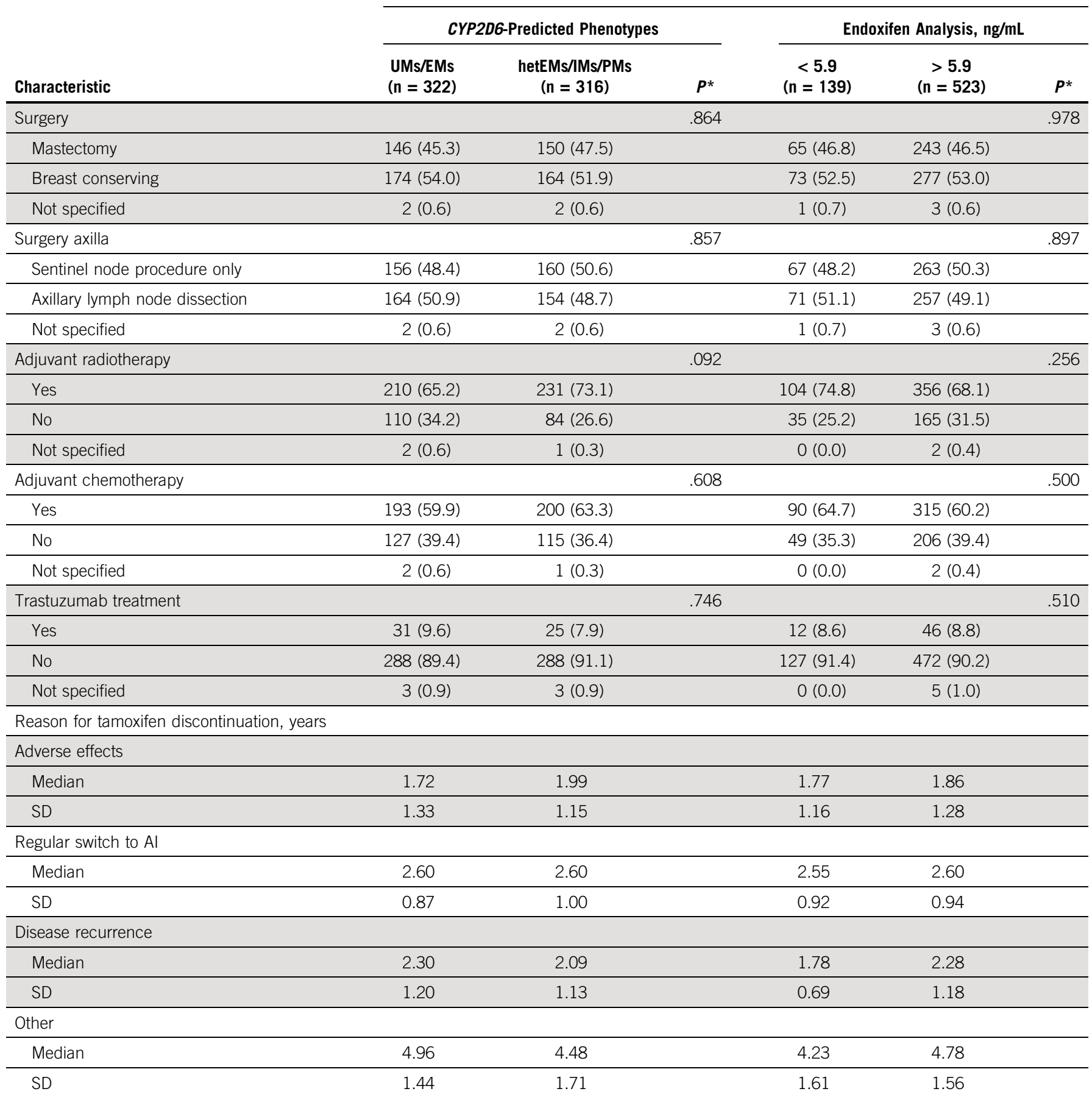

Abbreviations: Al, aromatase inhibitor; EM, extensive metabolizer; FISH, fluorescence in situ hybridization; HER2, human epidermal growth factor receptor 2; hetEM, heterozygous extensive metabolizer; IM, intermediate metabolizer; PM, poor metabolizer; SD, standard deviation; UM, ultra-metabolizer.

*In all cases, no missing data were included when comparing groups.

large number of patients, in which a small effect was observed, with CYP2D6 PMs being associated with worse survival (HR, $1.25 ; 95 \% \mathrm{Cl}, 1.06$ to $1.47 ; P=.009)$. Because of strict selection criteria and the exclusion of large studies such as the ATAC (Arimidex, Tamoxifen, Alone or in Combination) trial, ${ }^{26}$ the BIG (Breast International Group)
1-98 trial, ${ }^{27}$ and the TEAM (Tamoxifen and Exemestane in Early Breast Cancer) trial, ${ }^{28}$ this small effect has been questioned. At first, the ATAC and BIG 1-98 trials were ruled out because of the use of tumor as a source of DNA. Even so, a study by our group used tumor-derived DNA but rejected loss of heterozygosity by studying microsatellite 
TABLE 2. Cox Proportional Hazards Model of RFSt

\begin{tabular}{|c|c|c|c|c|c|c|c|}
\hline Endoxifen Analysis & No. of Patients & \multicolumn{3}{|c|}{ Univariable Analysis } & \multicolumn{3}{|c|}{ Multivariable Analysis* } \\
\hline Endoxifen, ng/mL (as continuous variable) & 662 & 0.989 & 0.945 to 1.035 & .627 & 0.991 & 0.946 to 1.038 & .691 \\
\hline$<5.9$ & 139 & 1.000 & Reference & & 1.000 & Reference & \\
\hline$>5.9$ & 523 & 1.426 & 0.673 to 3.021 & .354 & 1.538 & 0.719 to 3.290 & .267 \\
\hline Q1 $(<6.6)$ & 165 & 1.000 & Reference & .282 & 1.000 & Reference & .187 \\
\hline Q2 (6.6-10.3) & 167 & 1.820 & 0.851 to 3.891 & .123 & 1.986 & 0.909 to 4.340 & .085 \\
\hline Q3 (10.3-14.1) & 165 & 1.314 & 0.583 to 2.959 & .510 & 1.331 & 0.580 to 3.059 & .500 \\
\hline Q4 $(>14.1)$ & 165 & 0.977 & 0.415 to 2.302 & .957 & 0.950 & 0.399 to 2.262 & .907 \\
\hline
\end{tabular}

Abbreviations: EM, extensive metabolizer; hetEM, heterozygous extensive metabolizer; HR, hazard ratio; IM, intermediate metabolizer; PM, poor metabolizer; Q, quartile; RFSt, relapse-free survival (censored at the time of tamoxifen discontinuation); UM, ultra-metabolizer.

*Adjusted for human epidermal growth factor receptor $2 /$ neu status, histologic grade and classification, tumor size, and nodal stage.

markers flanking the CYP2D6 gene. ${ }^{28}$ Another major concern about the studies published is their retrospective nature, which may have introduced bias. In addition, none of these studies was specifically designed for the purpose of determining such an association, and this may have affected data retrieval.

Many studies have shown that low-activity CYP2D6 genotypes result in lower endoxifen concentrations compared with EMs. ${ }^{12}$ As a consequence, endoxifen concentrations have been postulated to be a predictor of tamoxifen efficacy. ${ }^{29}$ In a few retrospective studies, patients with low endoxifen concentrations were associated with worse survival outcomes. ${ }^{12-14}$ Madlensky et al ${ }^{12}$ suggested a threshold for endoxifen of $5.9 \mathrm{ng} / \mathrm{mL}$, above of which

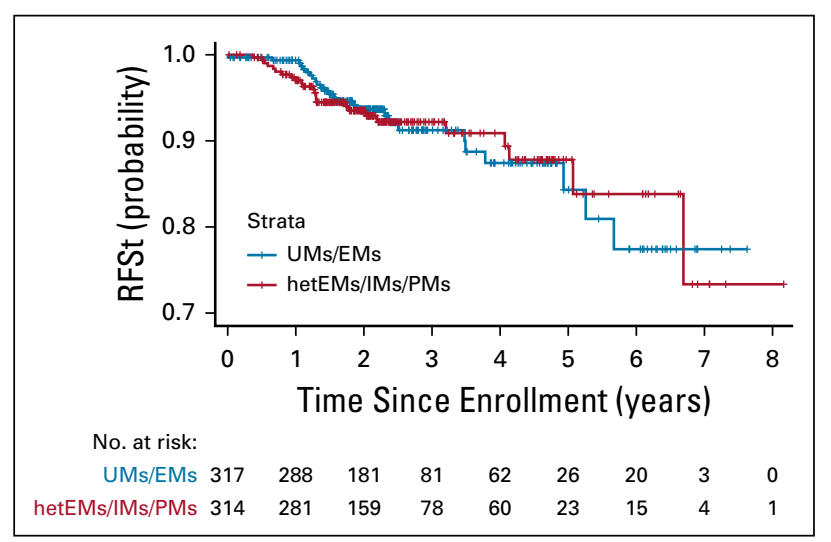

FIG 3. Kaplan-Meier curve for relapse-free survival (censored at the time of tamoxifen discontinuation; RFSt). EM, extensive metabolizer; hetEM, heterozygous extensive metabolizer; IM, intermediate metabolizer; PM, poor metabolizer; UM, ultra-rapid metabolizer. patients had at least a $26 \%$ lower chance of relapse (HR, $0.74 ; 95 \% \mathrm{Cl}, 0.55$ to 1.00 ). Following this approach, we also divided our cohort into two groups (below and above $5.9 \mathrm{ng} / \mathrm{mL}$ ). In our comparison, no significant differences were found. However, we also explored the possibility of dividing our cohort into quartiles, but again, no statistical associations were observed. In line with our results, a recent study analyzed the role of endoxifen therapeutic drug monitoring in the locally advanced and metastatic settings. ${ }^{30}$ Remarkably, the objective response rate in the lowest quartile $(0.7$ to $6.9 \mu \mathrm{g} / \mathrm{L})$ was not significantly different compared with that of the higher quartiles.

In this study, the sample size of 650 patients was based upon the studies of Goetz et $\mathrm{al}^{7}$ and Gonzalez-Santiago et al, ${ }^{19}$ and we assumed an HR of 2.0. Therefore, we cannot formally exclude a smaller effect $(H R<2.0)$ of CYP2D6 genotypes or endoxifen concentrations on clinical outcome.

In the last 10 years, Dutch guidelines have recommended a switch to an Al after 2 or 3 years of tamoxifen therapy for postmenopausal women. ${ }^{1,2}$ We are aware that the complete follow-up is relatively short, considering the possibility of disease recurrence in luminal breast cancer up to 20 years after diagnosis. ${ }^{31}$ In the CYPTAM study, in approximately $66.0 \%$ of patients, the possible detrimental effect of low endoxifen levels may have been weakened because of switching to an Al. However, because we censored patients at the time of tamoxifen discontinuation, potential effect modification by Al use was avoided, because only survival time during tamoxifen therapy was counted, and we could separate the effect of tamoxifen exposure from the effect of Al exposure. Along the same lines, we also examined RFSc, 
TABLE 3. Cox Proportional Hazards Model of RFSt: Univariable and Multivariable Analyses

Univariable Analysis

Multivariable Analysis*

Variable

No. of Patients

HR $\quad 95 \%$ Cl

$666 \quad 1.017 \quad 0.994$ to 1.040

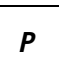

Tumor size

\begin{tabular}{ll}
\hline $\mathrm{T} 1$ \\
\hline $\mathrm{T} 2$ \\
$\mathrm{~T} 3 / \mathrm{T} 4$
\end{tabular}

Nodal status

\begin{tabular}{c}
\hline N0 \\
\hline N1 \\
\hline N2 \\
\hline N3 \\
\hline Grade \\
\hline 1
\end{tabular}

\begin{tabular}{|c|c|c|c|c|c|c|c|}
\hline 1 & 94 & 1.000 & Reference & .420 & 1.00 & Reference & .115 \\
\hline 2 & 378 & 0.899 & 0.409 to 1.977 & .792 & 0.621 & 0.275 to 1.404 & .252 \\
\hline 3 & 188 & 1.330 & 0.580 to 3.051 & .500 & 1.115 & 0.474 to 2.625 & .803 \\
\hline Negative & 605 & 1.000 & Reference & & 1.00 & Reference & \\
\hline Positive & 59 & 1.402 & 0.634 to 3.101 & .404 & 1.402 & 0.578 to 3.403 & .455 \\
\hline Ductal classification & 508 & 1.000 & Reference & & 1.000 & Reference & \\
\hline Lobular classification & 94 & 3.435 & 1.927 to 6.121 & $<.001$ & 4.015 & 2.014 to 8.003 & $<.001$ \\
\hline Other & 62 & 1.139 & 0.403 to 3.222 & .806 & 1.125 & 0.339 to 3.738 & .848 \\
\hline \multicolumn{8}{|l|}{ Progesterone status } \\
\hline Negative & 127 & 1.000 & Reference & & & & \\
\hline \multicolumn{8}{|l|}{ Surgery } \\
\hline Breast conserving & 352 & 1.523 & 0.879 to 2.640 & .134 & & & \\
\hline \multicolumn{8}{|l|}{ Surgery axilla } \\
\hline Sentinel node procedure & 333 & 1.00 & Reference & & & & \\
\hline Axillary lymph node dissection & 329 & 1.523 & 0.879 to 2.640 & .134 & & & \\
\hline \multicolumn{8}{|l|}{ Chemotherapy } \\
\hline No & 257 & 1.000 & Reference & & & & \\
\hline Yes & 407 & 1.084 & 0.613 to 1.916 & .781 & & & \\
\hline \multicolumn{8}{|l|}{ Radiotherapy } \\
\hline
\end{tabular}

Abbreviations: EM, extensive metabolizer; HER, human epidermal growth factor receptor; hetEM, heterozygous extensive metabolizer; HR, hazard ratio; IM, intermediate metabolizer; PM, poor metabolizer; Q, quartile; RFSt, relapse-free survival (censored at the time of tamoxifen discontinuation); UM, ultrametabolizer.

${ }^{*}$ Adjusted for HER2/neu status, histologic grade and classification, tumor size, and nodal stage. 
including during $\mathrm{Al}$ therapy and after stopping endocrine therapy, enabling the inclusion of later recurrences. This latter analysis is specifically useful, because it reflects the common practice of postmenopausal patients starting with tamoxifen and subsequently switching to an Al after 2 to 3 years of treatment. Therefore, whether these results could be extrapolated to other tamoxifen regimens remains uncertain, as does the impact of these results in the long term.

Menopausal status is clinically relevant but was not available for a majority of women in our study. To evaluate any influence of menopausal status on outcome, we used age group status as a proxy. Although correcting for menopausal status only slightly modified our results, we still failed to find an association between CYP2D 6 genotypes or endoxifen concentrations and clinical outcome.

A potential limitation of the study could be the study design, with a risk of selection bias resulting from the inclusion of patients who started with tamoxifen therapy up to 12 months before study enrollment. As a consequence, early recurrences may have been missed. However, the analysis shows that the association of RFSt, starting from the beginning of tamoxifen treatment, only slightly modified our results and did not yield a statistically significant association. Therefore, we believe selection bias is minimal. Similarly, the missed percentage of early recurrences is deemed low $(<1 \%) .{ }^{32}$ Indeed, our recurrence rate during the first year $(2.1 \%)$ is highly similar to those reported in larger studies, ${ }^{28,32}$ indicating a minimal effect of early recurrences. Moreover, 2 months of tamoxifen therapy is required to assure steady-state concentrations before the effect of tamoxifen can be evaluated, and therefore, any early recurrence before this time would be unlikely to be explained by tamoxifen exposure. Overall, we expect that selection bias as a result of the chosen study design was minimal and unlikely to have changed our results or conclusions. Also, no testing for tamoxifen adherence could possibly be a limitation, because it was not directly measured. Although data on tamoxifen adherence were

\section{AFFILIATIONS}

${ }^{1}$ Leiden University Medical Center, Leiden, the Netherlands

${ }^{2}$ Netherlands Cancer Institute/Antoni van Leeuwenhoek, Amsterdam, the Netherlands

${ }^{3}$ Alrijne Leiden, Leiden, the Netherlands

${ }^{4}$ Reinier de Graaf Gasthuis, Delft, the Netherlands

${ }^{5}$ Medical Center Alkmaar, Alkmaar, the Netherlands

${ }^{6}$ Zaans Medisch Centrum, Zaandam, the Netherlands

${ }^{7}$ Alrijne Leiderdorp, Leiderdorp, the Netherlands

${ }^{8}$ Katholieke Universiteit Leuven, Leuven, Belgium

${ }^{9}$ St Antonius Ziekenhuis, Nieuwegein, the Netherlands

${ }^{10}$ University Hospitals Leuven, Leuven, Belgium

\section{CORRESPONDING AUTHOR}

Henk-Jan Guchelaar, PharmD, PhD, Department of Clinical Pharmacy and Toxicology, Leiden University Medical Center, Albinusdreef 22300 RC Leiden, the Netherlands; e-mail: h.j.guchelaar@lumc.nl. lacking, information on persistence was well recorded. A comparison of mean endoxifen concentrations between patients who discontinued tamoxifen earlier because of adverse effects and those who did not stop did not significantly differ (adverse effects: $11.17 \mathrm{ng} / \mathrm{mL} v$ no adverse effects: $10.96 \mathrm{ng} / \mathrm{mL} ; P=.335$ ).

A potential limitation of this study could be the lack of accurate information on concomitant CYP2D6 inhibitor use during tamoxifen therapy. This concomitant use could bias the analysis between CYP2D6 genotypes and RFSt, because it could have led to misclassification of CYP2D6 phenotypes. However, the influence of potent CYP2D6 inhibitors was estimated to be low, because concomitant CYP2D6 inhibitors are infrequently prescribed in the Netherlands, ${ }^{33}$ especially in the last decade, because of greater awareness of the potential interactions among prescribers. According to the Clinical Pharmacogenetics Implementation Consortium Guideline for CYP2D6 and tamoxifen therapies, ${ }^{34}$ potent CYP2D6 inhibitors are contraindicated during tamoxifen therapy.

A more accurately predicted CYP2D6 phenotype by genotype and comedication is still merely a proxy for endoxifen concentration. When determining the relation between endoxifen concentrations and clinical outcomes, we are only interested in steady-state endoxifen concentrations, regardless of the cause of lower endoxifen concentrations (eg, genotype and concomitant CYP2D6 inhibitor use). The lack of information regarding comedication would be unlikely to have influenced the association between endoxifen concentrations and clinical outcomes.

In conclusion, the results of this prospective, specifically designed study do not support CYP2D6genotyping to guide tamoxifen treatment in the adjuvant setting. Similarly, our data do not justify therapeutic drug monitoring based on endoxifen concentrations in patients with breast cancer receiving tamoxifen.

\section{PRIOR PRESENTATION}

Presented at the Annual Meeting of the American Society of Clinical Oncology, Chicago, IL, June 1-5, 2018.

\section{SUPPORT}

Supported by ZOLEON (Stichting Oncologie Holland West) Project 09.09 grant.

AUTHORS' DISCLOSURES OF POTENTIAL CONFLICTS OF INTEREST AND DATA AVAILABILITY STATEMENT

Disclosures provided by the authors and data availability statement (if applicable) are available with this article at DOI https://doi.org/10.1200/ JCO.18.00307 


\section{AUTHOR CONTRIBUTIONS}

Conception and design: Vincent Dezentjé, Hans Gelderblom, Henk-Jan Guchelaar

Financial support: Hans Gelderblom

Administrative support: Anabel Sanchez-Spitman, Vincent Dezentjé, Lynn Jongen

Provision of study material or patients: Vincent Dezentjé, Jesse Swen, Carolien Smorenburg, Aart van Bochove, Anneke Zeillemaker, Lynn Jongen, Hans Gelderblom

Collection and assembly of data: Anabel Sanchez-Spitman, Vincent Dezentjé, Jesse Swen, Dirk Jan A.R. Moes, Erdogan Batman, Edith van Druten, Carolien Smorenburg, Aart van Bochove, Anneke Zeillemaker, Lynn Jongen, Maartje Los, Patrick Neven
Data analysis and interpretation: Anabel Sanchez-Spitman, Vincent Dezentjé, Jesse Swen, Dirk Jan A.R. Moes, Stefan Böhringer, Maartje Los, Patrick Neven, Hans Gelderblom, Henk-Jan Guchelaar Manuscript writing: All authors

Final approval of manuscript: All authors

Accountable for all aspects of the work: All authors

\section{ACKNOWLEDGMENT}

We thank Matthew Goetz, MD, from Mayo Clinic, Rochester, MN, for critically reading and commenting on the manuscript. We also thank Roche for providing the Amplichip P450 tests, Integraal Kankercentrum Nederland for data management, and ZOLEON for its grant.

\section{REFERENCES}

1. Burstein HJ, Temin S, Anderson H, et al: Adjuvant endocrine therapy for women with hormone receptor-positive breast cancer: American Society of Clinical Oncology clinical practice guideline focused update. J Clin Oncol 32:2255-2269, 2014

2. Senkus E, Kyriakides S, Ohno S, et al: Primary breast cancer: ESMO clinical practice guidelines for diagnosis, treatment and follow-up. Ann Oncol 26(Suppl 5): v8-v30, 2015

3. Knowledge Institute of Medical Specialists: Dutch Breast Cancer - Hormonal Therapy - Guideline - Richtlijnendatabase. https://richtlijnendatabase.nl/en/ richtlijn/breast_cancer/adjuvant_systemic_therapy/hormonal_therapy.html

4. Stearns V, Johnson MD, Rae JM, et al: Active tamoxifen metabolite plasma concentrations after coadministration of tamoxifen and the selective serotonin reuptake inhibitor paroxetine. J Natl Cancer Inst 95:1758-1764, 2003

5. Jin Y, Desta Z, Stearns V, et al: CYP2D6 genotype, antidepressant use, and tamoxifen metabolism during adjuvant breast cancer treatment. J Natl Cancer Inst 97:30-39, 2005

6. Sanchez-Spitman AB, Moes DA, Gelderblom H, et al: The effect of rs5758550 on CYP2D6*2 phenotype and formation of endoxifen in breast cancer patients using tamoxifen. Pharmacogenomics 18:1125-1132, 2017

7. Goetz MP, Rae JM, Suman VJ, et al: Pharmacogenetics of tamoxifen biotransformation is associated with clinical outcomes of efficacy and hot flashes. J Clin Oncol 23:9312-9318, 2005

8. Mürdter TE, Schroth W, Bacchus-Gerybadze L, et al: Activity levels of tamoxifen metabolites at the estrogen receptor and the impact of genetic polymorphisms of phase I and II enzymes on their concentration levels in plasma. Clin Pharmacol Ther 89:708-717, 2011

9. Borges S, Desta Z, Li L, et al: Quantitative effect of CYP2D6 genotype and inhibitors on tamoxifen metabolism: Implication for optimization of breast cancer treatment. Clin Pharmacol Ther 80:61-74, 2006

10. Hertz DL, Deal A, Ibrahim JG, et al: Tamoxifen dose escalation in patients with diminished CYP2D6 activity normalizes endoxifen concentrations without increasing toxicity. Oncologist 21:795-803, 2016

11. Dezentjé VO, Opdam FL, Gelderblom H, et al: CYP2D6 genotype- and endoxifen-guided tamoxifen dose escalation increases endoxifen serum concentrations without increasing side effects. Breast Cancer Res Treat 153:583-590, 2015

12. Madlensky L, Natarajan L, Tchu S, et al: Tamoxifen metabolite concentrations, CYP2D6 genotype, and breast cancer outcomes. Clin Pharmacol Ther 89: 718-725, 2011

13. Saladores P, Mürdter T, Eccles D, et al: Tamoxifen metabolism predicts drug concentrations and outcome in premenopausal patients with early breast cancer. Pharmacogenomics J 15:84-94, 2015

14. Helland T, Henne N, Bifulco E, et al: Serum concentrations of active tamoxifen metabolites predict long-term survival in adjuvantly treated breast cancer patients. Breast Cancer Res 19:125, 2017

15. Goetz MP, Ingle JN: CYP2D6 genotype and tamoxifen: Considerations for proper nonprospective studies. Clin Pharmacol Ther 96:141-144, 2014

16. The CYPTAM study: Effect of CYP2D6 genotype on pharmacokinetics and clinical outcome in tamoxifen treated breast cancer patients. http://www.trialregister. nl/trialreg/admin/rctview.asp?TC=1509

17. Schroth W, Goetz MP, Hamann U, et al: Association between CYP2D6 polymorphisms and outcomes among women with early stage breast cancer treated with tamoxifen. JAMA 302:1429-1436, 2009

18. Teunissen SF, Rosing H, Koornstra RH, et al: Development and validation of a quantitative assay for the analysis of tamoxifen with its four main metabolites and the flavonoids daidzein, genistein and glycitein in human serum using liquid chromatography coupled with tandem mass spectrometry. J Chromatogr B Analyt Technol Biomed Life Sci 877:2519-2529, 2009

19. Gonzalez-Santiago S, Zárate R, Haba-Rodríguez J, et al: CYP2D6*4 polymorphism as blood predictive biomarker of breast cancer relapse in patients receiving adjuvant tamoxifen. J Clin Oncol 25, 2007 (suppl; abstr 590)

20. Dmitrienko A, Tamhane AC: Gatekeeping procedures with clinical trial applications. Pharm Stat 6:171-180, 2007

21. Dmitrienko A, Tamhane AC, Wang X, et al: Stepwise gatekeeping procedures in clinical trial applications. Biom J 48:984-991, 2006

22. Bradford LD: CYP2D6 allele frequency in European Caucasians, Asians, Africans and their descendants. Pharmacogenomics 3:229-243, 2002

23. van de Velde CJ, Rea D, Seynaeve C, et al: Adjuvant tamoxifen and exemestane in early breast cancer (TEAM): A randomised phase 3 trial. Lancet 377 : 321-331, 2011

24. Chen L, Linden HM, Anderson BO, et al: Trends in 5-year survival rates among breast cancer patients by hormone receptor status and stage. Breast Cancer Res Treat 147:609-616, 2014

25. Province MA, Goetz MP, Brauch H, et al: CYP2D6 genotype and adjuvant tamoxifen: meta-analysis of heterogeneous study populations. Clin Pharmacol Ther 95:216-227, 2014

26. Rae JM, Drury S, Hayes DF, et al: CYP2D6 and UGT2B7 genotype and risk of recurrence in tamoxifen-treated breast cancer patients. J Natl Cancer Inst 104: 452-460, 2012 
27. Regan MM, Leyland-Jones B, Bouzyk M, et al: CYP2D6 genotype and tamoxifen response in postmenopausal women with endocrine-responsive breast cancer: The Breast International Group 1-98 trial. J Natl Cancer Inst 104:441-451, 2012

28. Dezentjé VO, van Schaik RH, Vletter-Bogaartz JM, et al: CYP2D6 genotype in relation to tamoxifen efficacy in a Dutch cohort of the tamoxifen exemestane adjuvant multinational (TEAM) trial. Breast Cancer Res Treat 140:363-373, 2013

29. Binkhorst L, Mathijssen RH, Jager A, et al: Individualization of tamoxifen therapy: Much more than just CYP2D6 genotyping. Cancer Treat Rev 41:289-299, 2015

30. Neven P, Jongen L, Lintermans A, et al: Tamoxifen metabolism and efficacy in breast cancer: A prospective multicentre trial. Clin Cancer Res 24:2312-2318, 2018

31. Pan H, Gray R, Braybrooke J, et al: 20-year risks of breast-cancer recurrence after stopping endocrine therapy at 5 years. N Engl J Med 377:1836-1846, 2017

32. Early Breast Cancer Trialists' Collaborative Group (EBCTCG): Aromatase inhibitors versus tamoxifen in early breast cancer: Patient-level meta-analysis of the randomised trials. Lancet 386:1341-1352, 2015

33. Dezentjé VO, van Blijderveen NJ, Gelderblom H, et al: Effect of concomitant CYP2D6 inhibitor use and tamoxifen adherence on breast cancer recurrence in early-stage breast cancer. J Clin Oncol 28:2423-2429, 2010

34. Goetz MP, Sangkuhl K, Guchelaar HJ, et al: Clinical Pharmacogenetics Implementation Consortium (CPIC) guideline for CYP2D6 and tamoxifen therapy. Clin Pharmacol Ther 103:770-777, 2018 


\section{Tamoxifen Pharmacogenetics and Metabolism: Results From the Prospective CYPTAM Study}

The following represents disclosure information provided by authors of this manuscript. All relationships are considered compensated. Relationships are self-held unless noted. I = Immediate Family Member, Inst = My Institution. Relationships may not relate to the subject matter of this manuscript. For more information about ASCO's conflict of interest policy, please refer to www.asco.org/rwc or ascopubs.org/jco/site/ifc.

Dirk Jan A.R. Moes

Research Funding: Chiesi Pharma (Inst)

Carolien Smorenburg

Travel, Accommodations, Expenses: Roche

Lynn Jongen

Research Funding: Puma Biotechnology

Patrick Neven

Consulting or Advisory Role: Novartis (Inst), Eli Lilly (Inst), Pfizer (Inst), Ipsen (Inst)

Travel, Accommodations, Expenses: Pfizer, Roche

\section{Hans Gelderblom}

Patents, Royalties, Other Intellectual Property: Amgen (Inst), BoehringerIngelheim (Inst), Pfizer (Inst), Novartis (Inst), Pharmamar (Inst), Daiichi Sankyo (Inst), Five Prime (Inst)

Other Relationship: Daiichi Sankyo

No other potential conflicts of interest were reported. 
Collaborating hospitals and local investigators: Leiden University Medical Center, Gelderblom and V.O. Dezentjé; Alrijne Leiden, R. Vree and E. Batman; Reinier de Graaf Groep, E.M. Maartense; Noordwest Ziekenhuisgroep, C. Smorenburg; St Antonius Ziekenhuis, M. Los; Rijnland Ziekenhuis, A.M. Zeillemaker; Zaans Medisch Centrum, A. Van Bochove; Waterland Ziekenhuis, J.A.C. Brakenhoff; IJsselland Ziekenhuis, D.F.S. Kehrer; Spaarne Gasthuis, P. Kuijer; Meander Medisch Centrum, H.J. Bloemendal; Tergooi Ziekenhuizen, S.A. Luijkx; HMC Bronovo Ziekenhuis, N.I. Weijl; Langeland Ziekenhuis, Goossens; Diakonessenhuis Utrecht, D. Ten Bokkel Huinink; HAGA Ziekenhuis, J.W.S. Merkus; St Antonius Ziekenhuis, M.J. Verhaar; Groene Hart Ziekenhuis, A. Van der Torren; Amstelland Ziekenhuis, A H. Baan; and UZ Leuven (also on behalf of the other Belgian hospitals), P. Neven.

\section{Tamoxifen and Metabolite Measurements and CYP2D6 Genotyping}

Steady-state trough levels of tamoxifen and its metabolites were measured with high-performance liquid chromatography-tandem mass spectrometry. ${ }^{18}$

The considered CYP2D6-predicted phenotypes are: ultra-rapid (duplication of fully active alleles), extensive (with two fully active alleles, nowadays indicated as normal metabolizers), heterozygous extensive (with one fully active allele and one nonactive allele), intermediate (with two low activity alleles or a combination of one low activity allele and one inactive allele), and poor metabolizer (with two inactive alleles). Alleles with decreased CYP2D6 activity were ${ }^{*} 9,{ }^{*} 10,{ }^{*} 17,{ }^{*} 29,{ }^{*} 36$, ${ }^{*} 41,{ }^{*} 10 \times N,{ }^{*} 17 \times N$, and ${ }^{*} 41 \times N$, whereas CYP2D6 inactive alleles were ${ }^{*} 3,{ }^{*} 4,{ }^{*} 5,{ }^{*} 6,{ }^{*} 7,{ }^{*} 8,{ }^{*} 11,{ }^{*} 14 \mathrm{~A},{ }^{*} 15,{ }^{*} 19,{ }^{*} 20,{ }^{*} 40$, and ${ }^{*} 4 x N$.

\section{Statistical Methods and Analysis}

The distribution of the relapse-free survival (censored at the time of tamoxifen discontinuation) end point was estimated overall using Kaplan-Meier analysis, whereas differences between CYP2D6 genotypes and endoxifen concentrations were assessed by a log-rank test. Fisher's exact test was used to evaluate baseline characteristics between CYP2D6 phenotype groups and endoxifen groups. To evaluate differences in allele frequencies of CYP2D 6 and deviations from Hardy-Weinberg equilibrium, $\chi^{2}$ tests were carried out.

Cox regression analyses were performed in two steps. First, a univariable analysis was carried out, and then a multivariable analysis was performed. Only covariables with $P<.1$ in the univariable analysis were included in the multivariable analysis. In any case, histologic grade, tumor size, nodal status, and human epidermal growth factor receptor $2 /$ neu status were used in the multivariable analysis because of their known clinical relevance.

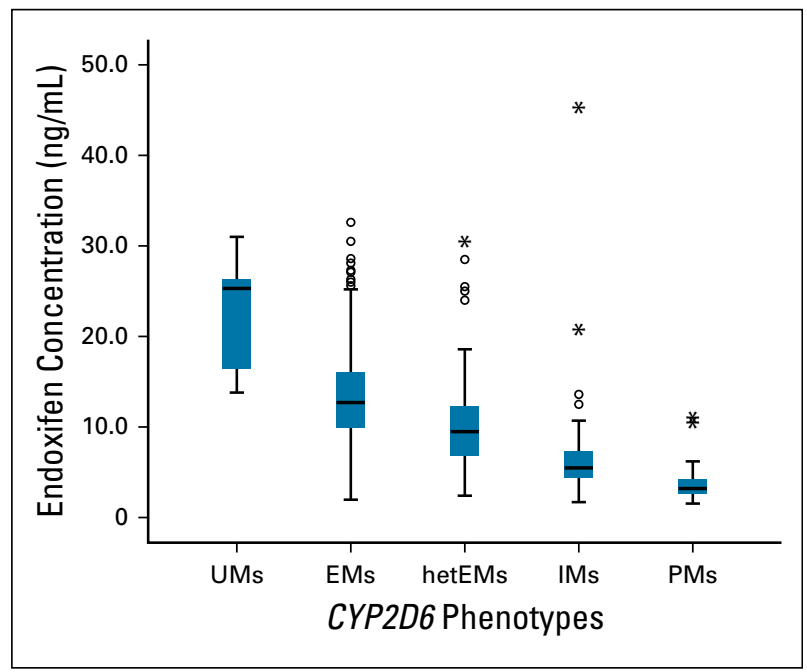

FIG A1. Endoxifen concentration in CYP2D6 phenotypes. ( ${ }^{*}$ ) A total of $42.3 \%$ of the variance of endoxifen serum concentration is explained by CYP2D6-predicted phenotypes. EMs, extensive metabolizers; hetEMs, heterozygous extensive metabolizers; IMs, intermediate metabolizers; PMs, poor metabolizers; UMs, ultra-metabolizers. 